\title{
Conceito, configuração e aplicação de um simulador de direção no Brasil - Estudo de caso
}

\author{
Maria Izabel dos Santos ${ }^{1}$, Paulo Tadeu Meira e Silva de Oliveira' ${ }^{2}$, Rogério Lemos Ribeiro ${ }^{3}$, Ana \\ Paula Camargo Larocca ${ }^{4}$, Felipe Issa Kabbach Junior ${ }^{5}$
}

'Escola de Engenharia de São Carlos, USP, São Paulo, Brasil, mis@usp.br ${ }^{2}$ Escola de Engenharia de São Carlos, USP, São Paulo, Brasil, poliver@usp.br ${ }^{3}$ Faculdade de Engenharia Civil, UFU, Minas Gerais, Brasil, rogerio.ribeiro@ufu.br 4Departamento de Engenharia de Transportes, EESC - USP, São Paulo, Brasil, larocca.ana@usp.br ${ }^{5}$ Departamento de Engenharia de Transportes, EP-USP, São Paulo, Brasil, felipe.kabbach@poli.usp.br

\section{Recebido:}

21 de junho de 2016

Aceito para publicação:

29 de março de 2017

Publicado:

31 de agosto de 2017

Editor de área:

Flávio Cunto, UFC

\section{Palavras-chaves:}

Simulador de direção.

Segurança viária.

Análise estatística.

Comportamento condutor.

\section{Keywords:}

Driving Simulator.

Road safety.

Statistical analysis.

Driver's behavior.

DOI:10.14295/transportes.v25i2.1174

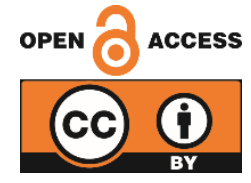

\begin{abstract}
RESUMO
Desde 2011, o mundo vive a Década de Ação para a Segurança Rodoviária (2011-2020) cujo objetivo é reduzir pela metade o número de vítimas de acidentes de trânsito. Simuladores de condução podem ajudar nos estudos sobre segurança viária sem oferecer risco aos voluntários e pesquisadores. O presente artigo descreve um simulador de médios porte e custo, utilizado principalmente por indústrias automotivas. Tais simuladores têm sido utilizados em diversas aplicações como estudos do comportamento de condutores, projeto geométrico de vias e sinalizações horizontal e vertical da pista. O simulador descrito possui características e desempenho similares ao de um veículo compacto real e os programas utilizados para compilação dos dados de entrada trabaIham em tempo real. Dois estudos conduzidos no simulador são apresentados de forma sucinta: o primeiro analisa a percepção dos condutores com relação à sinalização de um trecho de rodovia brasileira e o segundo avalia a alteração da capacidade cognitiva de um condutor quando submetido a uma tarefa secundária à da condução.
\end{abstract}

\begin{abstract}
Since 2011, the world is living a Decade of Action for Road Safety. The goal is to reduce the number of deaths and injuries by half which are occurring due to road traffic accidents. Driving simulators can help in road safety studies without offering any risk to researchers and volunteers. Current article describes a medium sized and cost efficient driving simulator, mainly used by automotive industries. Such simulators have been used in several other applications. The examples are studies related to: i) driver's behavior, ii) geometric track designs and iii) vertical or horizontal signage. Driving simulator has characteristics and performance similar to a compact vehicle. Software used to compile input data works in real time. At the end, two case studies using the driving simulator are presented. The first study is an assessment of the driver's perception regarding the signage along a stretch of a Brazilian highway. The second one evaluates the change in the cognitive capacity of the driver when he has a secondary task, to be performed simultaneously to the driving task.
\end{abstract}

\section{INTRODUÇÃO}

Com 1,25 milhões de mortes registradas anualmente devido a acidentes de trânsito (WHO | World Health Organization, 2015), a Organização Mundial da Saúde (OMS) lançou a Década de Ação pela Segurança no Trânsito (2011-2020). A meta é reduzir pela metade o número de vítimas fatais e lesões relacionadas aos acidentes de trânsito em todo o mundo. Ainda, segundo a OMS, para que o número de acidentes seja reduzido, devem ser trabalhados cinco pontos: (1) Desenvolvimento de planos estratégicos e metas factíveis em âmbito nacional; (2) Atuação conjunta entre setores público e privado; (3) Engajamento político, associado à alocação de recursos adequados; (4) Desenvolvimento adequado de políticas, pesquisas 
e atuações tanto no setor privado quanto no público; e (5) Coleta e disponibilidade de dados de boa qualidade (WHO | World Health Organization, 2015).

A coleta de dados de boa qualidade é um desafio muitas vezes negligenciado, já que a coleta de informações sobre acidentes depende de relatos dos envolvidos e de testemunhas, além da experiência do perito que analisa a cena do acidente. Essa condição insere erros e desvios de percepção do que de fato ocorreu (Evgenikos et al., 2010). Além disso, a reprodução de acidentes em campo para validação de teorias é algo inviável pelo alto risco da situação.

Uma solução cada vez mais explorada por pesquisadores é a utilização de modelos matemáticos para estudar os fatores que determinam a ocorrência de acidentes. 0 salto computacional ocorrido nas últimas décadas permitiu o desenvolvimento de programas capazes de resolver, em tempo real, as equações de movimento e força descritas por tais modelos matemáticos. A combinação de programas e equipamentos compõe os chamados simuladores de direção. Para que um simulador gere resultados significativos, em comparação a dados obtidos em campo, deve-se ter modelos que sejam representativos. Balci (1994) define modelo como sendo uma representação de um sistema, conceito ou problema. Modelos e simuladores são dependentes de dados de entrada, por isso desvios observados em tais dados e/ou hipóteses mal formuladas resultarão em perda de confiança nos resultados obtidos, ou seja, resultarão em maiores desvios.

Simuladores de direção se tornaram uma ferramenta amplamente utilizada para examinar o impacto das diferenças individuais de projetos de rodovias, condutores, comportamentos veiculares e a eficácia de alterações voltadas à segurança viária (Boyle e Lee, 2010 e Jamson et al., 2010). Em um estudo de análise comparativa de percepção de risco, Underwood et al (2011) mostram que, apesar das diferenças entre os ambientes virtual e real, é possível correlacionar os resultados de percepção de risco do condutor em um simulador com a situação real.

Rumschlag (2015) utilizou um simulador de direção de base fixa para estudar os efeitos de digitar mensagens em celulares durante a condução. Vinte e três homens e vinte e sete mulheres com idade média de 34,5 anos participaram do estudo. 0 estudo foi dividido em quatro fases: duas de condução no simulador e duas de questionário. A principal variável dependente era o desvio de percurso ocorrido durante o trajeto. A medida foi feita por meio de três variáveis independentes: porcentagem de pessoas que desviaram o percurso; número de desvios de percurso; e porcentagem do tempo de mensagens de texto no desvio de percurso. Quando não submetidos à conversa via mensagem, os participantes não registraram desvio de percurso. Porém, $66 \%$ dos participantes desviaram de trajetória enquanto enviavam mensagem de texto. 0 estudo também analisou os dados agrupando-os de acordo com o gênero e a idade do condutor, a duração da tarefa de envio de mensagem, o nível de habilidade de digitação e o histórico de uso de mensagens de texto (\#mensagens/semana).

Helland (2013) conduziu um estudo em simulador de direção com base fixa para definir e validar um método para avaliar a condução sob o efeito de drogas. Esse estudo foi feito com vinte voluntários do sexo masculino. Os testes foram conduzidos com pessoas sóbrias e com pessoas com concentração de álcool no sangue entre $0,5 \mathrm{~g} / \mathrm{L}$ e $0,9 \mathrm{~g} / \mathrm{L}$. 0 efeito placebo também foi introduzido no estudo como confundidor. A principal variável medida foi o desvio padrão da posição lateral (DPPL, "zigzag"). A maioria dos participantes mostrou um comportamento consistente entre os testes no simulador e os testes na via de teste. Entretanto, a falta de sensação de risco sentida pelo condutor pode ter influenciado alguns resultados do estudo, segundo os pesquisadores.

Um simulador de direção precisa de validação relativa satisfatória para ser considerada uma ferramenta útil (Törnros, 1998). Diversos estudos mostram que os simuladores são ferramentas efetivas para estudos de velocidade de condução (Bella, 2007, Godley et al., 2002, Bella e Calvi, 2013): para avaliar comportamento dos condutores em condições climáticas específicas (Broughton et al., 2007); para avaliar a segurança viária em intersecções sinalizadas (Yan et al., 2008); para entender a influência de drogas e álcool (Lenné et al., 2010, Helland et al., 2013) e para entender a influência de desordens mentais na condução (Devlin et al., 2012). Entretanto, não há consenso sobre a real fidelidade dos simuladores, 
pois eles podem apresentar deficiências que impactem negativamente os resultados, dependendo da aplicação.

Os simuladores podem ser classificados de acordo com sua aplicação (simuladores de treinamento e de pesquisa) ou de acordo com seu custo (baixo, médio e alto custo) (Blana, 1996b). Em ambas as classificações, as diferenças entre os modelos de simuladores estão tanto no equipamento que os compõe quanto nos programas. São os equipamentos que fazem a interface direta com o condutor, pois são os comandos (como volante, pedaleira, câmbio, tela, televisores e equipamentos de som) utilizados pelo condutor para dirigir. Os programas, por sua vez, são responsáveis pelo equacionamento e solução dessas equações segundo as entradas impostas. De acordo com as combinações entre equipamento e programas, os simuladores são classificados em baixo, médio e grande porte. Quanto mais complexo e mais fiel à realidade, maiores são os custos envolvidos para construção do simulador.

\section{DESCRIÇÃO DO SIMULADOR DE DIREÇÃO}

Um simulador de direção consiste basicamente em um condutor sentado em um ambiente que reproduz as condições de uso de um veículo incluindo controles normais como volante, embreagem e pedais de acelerador e freio. As ações do condutor e a posição do veículo virtual são continuamente calculadas por programas específicos e projetadas em tempo real em telas ou monitores. 0 cenário da rodovia, por sua vez, pode ser criado a partir de processamento digital do arquivo de projeto geométrico de uma via (existente ou idealizada), ou, ainda, a partir de informações coletadas in loco como latitude, longitude e inclinações. $O$ entorno da via também pode ser inserido no ambiente visualizado pelo condutor.

Os simuladores permitem que sejam avaliados, simultaneamente, fatores que compõem o sistema de trânsito. Por ser um ambiente virtual, é seguro tanto para o condutor quanto para o pesquisador (Lee $e t$ al., 2003) e permite pesquisar níveis que conduzam a situações de grande risco. Diversos estudos foram realizados para validação de simuladores em diferentes aplicações (Blana, 1996; Chan et al., 2010; Underwood et al., 2011; Knapper et al., 2015). Por meio do uso de simuladores, é possível o levantamento de informações variadas sem custos adicionais. Essas informações podem ser simples como a contagem do número de acidentes em um determinado trecho, ou podem ser complexas, envolvendo pós-processamento. A aplicação de simuladores de direção em estudos voltados para a segurança viária ainda é pouco explorado no Brasil em comparação aos países do hemisfério norte.

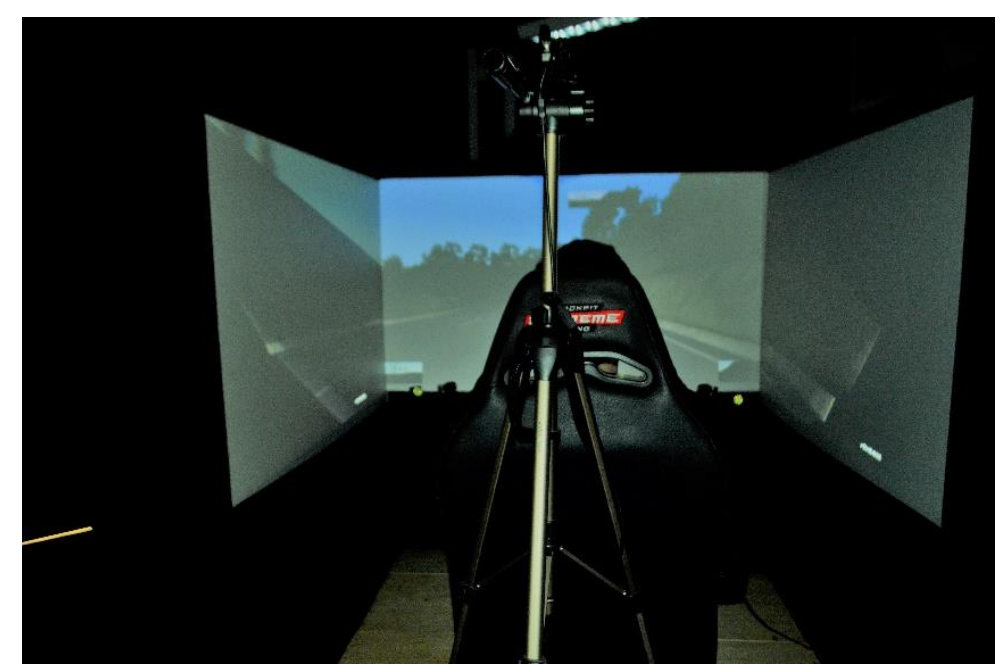

Figura 1. Simulador de direção

O presente trabalho discute a construção de um simulador de direção no Brasil. 0 simulador é de porte e custo médios, com base fixa, e utiliza telas de projeção para simular o ambiente de condução (Figura 1). 0 diagrama de blocos da Figura 2 representa a estrutura do simulador. Basicamente, pode-se dividi-lo em duas partes: equipamentos e programas. 
Apesar do simulador de base estática influenciar a velocidade percebida pelo condutor (Reymond et al., 2001), os experimentos conduzidos no simulador são comparativos, o que reduz a significância da aceleração lateral nas conclusões observadas. A ausência da aceleração lateral altera o tempo de reação do condutor a perturbações externas, como curvas, e diminui as margens de segurança com as quais o condutor trabalha (Kemeny e Panerai, 2003). Sendo assim, em estudos avançados, principalmente sobre comportamento do condutor, a utilização de um simulador de base móvel é mais adequada.

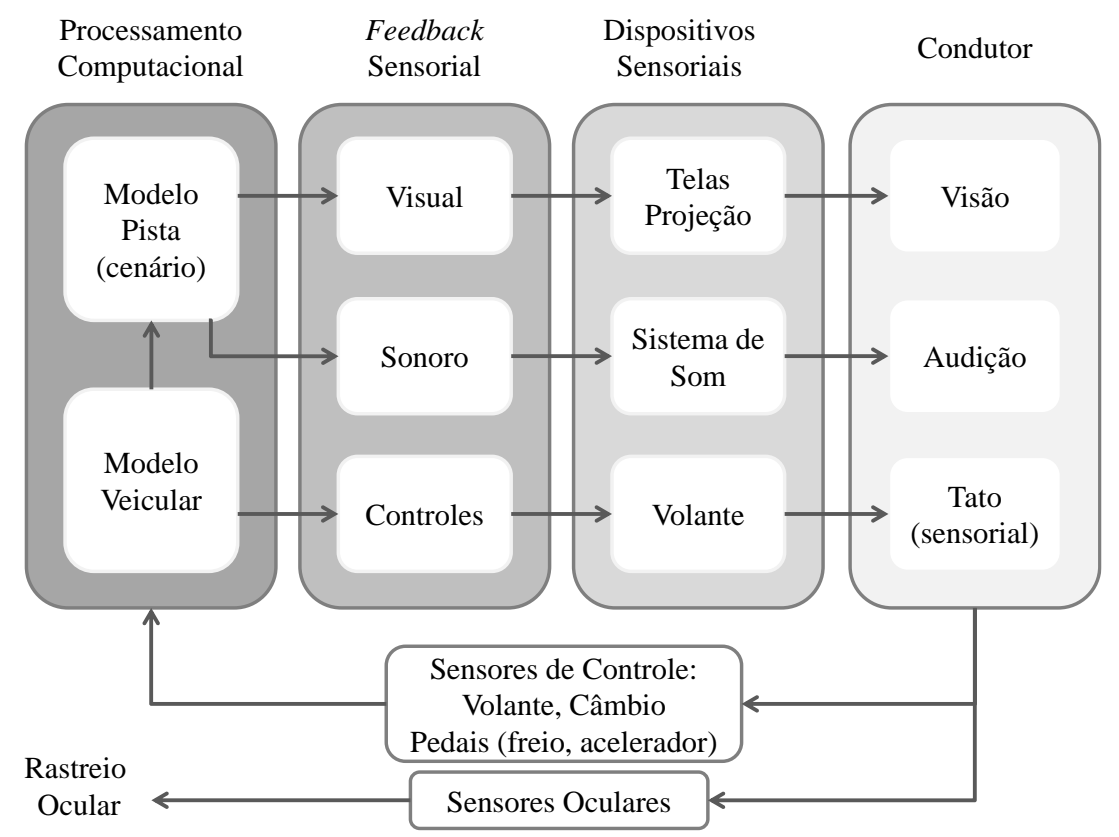

Figura 2. Estrutura do simulador de direção (Adaptado de Rangel, 2015)

\subsection{Equipamentos}

O processamento computacional é feito com três computadores de mesa acompanhados de monitores de 27", teclado e mouse. 0 primeiro computador (onde as análises da dinâmica veicular são calculadas em tempo real) tem processador Intel Core I7, com memória RAM DDR3 de 8.0 GB, HD com 2TB W.D, uma placa SVGA PCI EX com 3GB GTX780REF EVGA DDR5 384 bits e Sistema operacional Windows 8.1 ${ }^{\circledR}$. 0 segundo computador (utilizado para geração dos cenários) possui processador Intel Core I7, com memória RAM DDR3 de 8.0 GB, HD com 2TB W.D, duas placas SVGA PCI EX com 3GB GTX780REF EVGA DDR5 384 bits e Sistema Operacional Ubuntu 12.04 LTS. Já o terceiro computador (utilizado pelo sistema de rastreio ocular) possui processador Intel Core I7, com memória RAM DDR3 de 8.0 GB, HD com 1TB, placa mãe ASUS B85M-E e Sistema Operacional Windows $8.1^{\circledR}$.

O principal dispositivo de entrada do simulador é uma estação completa de pilotagem da marca Cockpit Extreme Racing ${ }^{\circledR}$, modelo XT Premium. 0 dispositivo possui um controle Logitech ${ }^{\circledR}$, modelo G27 composto por volante com feedback de força, manete de câmbio e pedaleira com pedais de freio, acelerador e embreagem. A estação contém, ainda, uma cadeira automotiva com regulagem de distância entre o banco e o volante. Também é possível fazer a regulagem de altura do volante. Esse equipamento é a interface direta do condutor com as modelagens veicular e do ambiente (rodovia e entornos).

Para aprimorar a sensação de realidade do condutor, duas caixas de som da marca Thonet et Vander ${ }^{\circledR}$, modelo Vertrag reproduzem sons como ruídos gerados pelo motor do veículo, chuva e vento. As caixas possuem 32 watts RMS de potência. Os sons referentes ao funcionamento do motor são ruídos reais de um motor similar ao do modelo do veículo.

Três projetores DepthQ HDs3D2, com resolução de 1080 p e taxa de projeção de $60 \mathrm{~Hz}$ são utilizados para projeção do ambiente virtual. As projeções são feitas em três telas planas de 1,4 m de largura e $0,8 \mathrm{~m}$ de altura. As telas formam $90^{\circ}$ entre si. A tela central possui resolução de $1,70 \mathrm{arcmin} / \mathrm{pixel}$. Essa 
configuração permite que o condutor tenha um amplo campo de visão do cenário, com, aproximadamente, $180^{\circ}$ na horizontal e $50^{\circ}$ na vertical.

Para o rastreio ocular, foi acoplado ao simulador um equipamento próprio para a aplicação: Smart Eye, modelo Pro 5.10 ${ }^{\circledR}$ (SE). O SE fornece dados brutos de confiança e dados filtrados em 3D. É capaz de rastrear remotamente direção do olhar, posição da cabeça, abertura de pálpebra, piscadas, pontos de fixação, tamanho da pupila, dentre outros monitoramentos e medições. 0 equipamento é capaz de detectar a interseção do olhar do condutor com objetos criados no ambiente virtual como placas de sinalização e pedestres. Os dados de saída são selecionáveis, ou seja, somente os dados de interesse do pesquisador são incluídos no arquivo e a maioria dos dados é transferida em tempo real. Por utilizar um sistema de coordenadas globais, a transformação para outro sistema de coordenadas é simples.

0 rastreio de cada olho pode ser feito separadamente, permitindo rastreamentos monoculares e binoculares. Os algoritmos utilizados são capazes de trabalhar tanto com rastreio da íris quanto da pupila. Diferentemente de outros equipamentos, pode ser utilizado para rastreamento de pupilas escuras ou brilhantes, e isso aumenta a gama de voluntários que podem ser utilizados sem necessidade da aquisição de equipamentos diferentes.

0 modelo Pro 5.10 é composto por três câmeras frontais que fazem o rastreio ocular. Uma câmera adicional posterior registra as cenas vistas pelo condutor (Erro! Fonte de referência não encontrada.Figura 3). Apesar de possuir instalações e calibrações mais complexas, a opção por três câmeras em vez de uma única objetiva conseguir maior precisão e é mais adequada quando se trata de estudos investigativos (Ahlström et al., 2010).

Um estudo recente, conduzido na Suécia, utilizou o SE para examinar o impacto da sonolência sobre os sintomas oculares após condução em simulador de direção (Filtness et al., 2017). As principais informações coletadas com o equipamento e utilizadas por Filtness foram número de piscadas, duração média e duração mediana das piscadas. Outro estudo, que também fez uso do SE, desenvolveu um método para prever as avaliações dos condutores sobre o nível de aceitabilidade de um aviso emitido em resposta a acidentes, quase acidentes e outros incidentes (Källhammer et al., 2007).

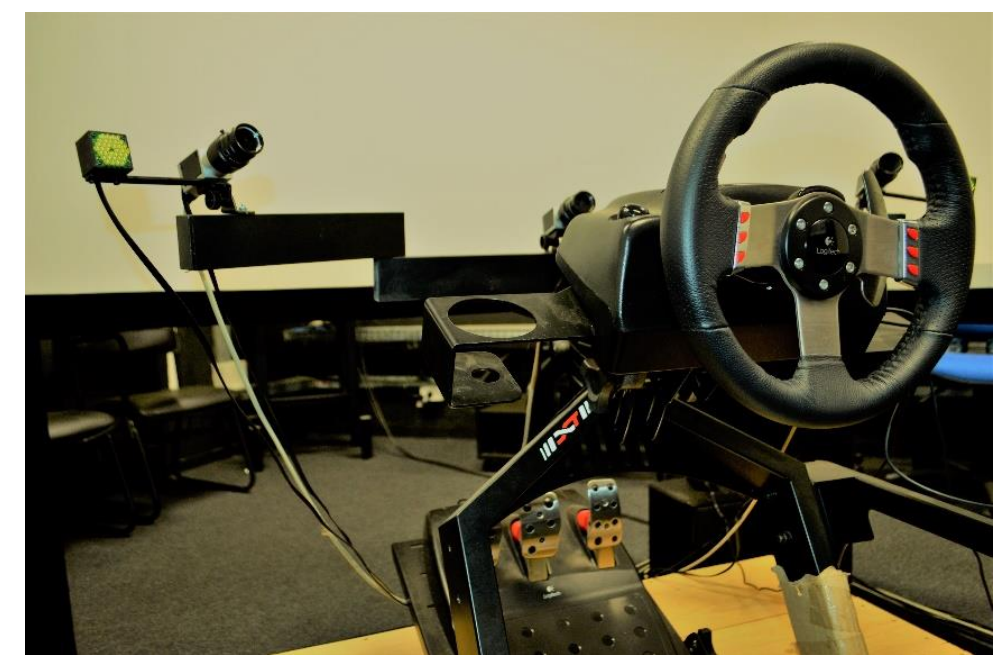

Figure 3. Equipamento utilizado para rastreio ocular: Smart Eye ${ }^{\circledR}$ modelo Pro 5.10

Os equipamentos estão acomodados em uma sala preta, climatizada com condicionador de ar para garantir que a temperatura do cômodo se mantenha adequada para voluntários e equipamentos. Possíveis enjoos devido ao uso do simulador podem ser evitados mantendo a sala climatizada com temperatura de $20^{\circ} \mathrm{C}$ (Fisher et al., 2011).

Os equipamentos de interface direta com o condutor estão fixados uma base estática de madeira de 1,4 m de comprimento, 1,3 m largura e 0,2 $\mathrm{m}$ de altura. As medidas foram definidas com base na altura 
da tela até o solo e na área necessária para acomodar os equipamentos (estação de pilotagem e equipamentos de som e de rastreio ocular).

\subsection{Programas}

Além dos sistemas operacionais descritos no item 2.1, são utilizados três programas específicos: Virtual Test Drive ${ }^{\circledR}$, Mapps $^{\circledR}$ e CarReal Time ${ }^{\circledR}$.

Para a geração de cenário e tráfego, foi utilizado o Virtual Test Drive (VTD ${ }^{\circledR}$ ). 0 programa é desenvolvido e comercializado pela empresa Vires ${ }^{\circledR}$. A alta qualidade do cenário gerado, a possibilidade de inserção de tráfego e a capacidade de trabalhar com simulações em tempo real foram decisivas para sua seleção. Tal programa possui uma cadeia completa de ferramentas para simuladores de direção, dentre as quais se destacam: ferramentas para geração de estradas, cenários e simulação de tráfegos, simulação de sons e geração de imagens em tempo real. Sua principal aplicação é na indústria automotiva. 0 VTD ${ }^{\circledR}$ é dividido em seis módulos: ROD, onde é gerada a malha rodoviária; v-SCENARIO e v-TRAFFIC, editores gráficos para monitoramento e definição dos cenários de tráfego - incluindo pedestres; v-IG, gerador de imagens em tempo real; v-IOS, interface gráfica com o usuário para gerenciamento das simulações; vTaskControl, estação de controle central que controla todos os fluxos de dados; e SOUND, inserção dos sons específicos de veículo para aumentar o grau de imersão do usuário.

O Mapps ${ }^{\circledR}$ é o programa utilizado para análise dos dados gerados pelo SE. Sua interface com o usuário é intuitiva e amigável. Ele permite análise em tempo real e no modo de pós-processamento, além de exportar dados em formato compatível com Microsoft Excel ${ }^{\circledR}$. Algumas de suas ferramentas são de grande uso para pesquisas com monitoramento ocular, como visualização gráfica das regiões de interesse para o rastreio ocular e visualização do rastro ocular e das fixações durante o monitoramento.

A modelagem veicular foi realizada utilizando o programa VI-CarRealTime ${ }^{\circledR}$ v.16 (CRT). Esse programa é a principal ferramenta utilizada atualmente pelas engenharias da indústria automotiva e por equipes de competições profissionais e amadoras para projeto e ajustes da dinâmica veicular. Cada subsistema do veículo é modelado separadamente e possui templates que auxiliam na modelagem. Assim como os demais programas, o CRT trabalha em tempo real.

Os programas selecionados fazem parte de uma solução comercializada pela empresa VI-Grade ${ }^{\circledR}$. Tal configuração aqui apresentada não é a única disponível, entretanto o critério para a seleção dos programas utilizados foi a melhor relação custo x benefício.

\subsection{Limitações do simulador de direção}

Apesar da capacidade de processamento em tempo real, de transmitir aos condutores estímulos similares aos reais e da alta qualidade das imagens geradas, é importante ressaltar as limitações observadas. Base fixa: a não transmissão de movimentos afeta a percepção de velocidade do condutor. Outro ponto negativo é a confusão causada ao sistema vestibular do condutor devido à divergência entre movimento observado (proveniente da condução em ambiente virtual) e movimento percebido (acelerações laterais, verticais e longitudinais sentidas quando em uma condução real ou em base móvel). Essa divergência pode causar enjoos em algumas pessoas.

Resolução visual: em comparação ao olho humano, o simulador de direção possui baixos contraste, resolução e luminosidade, já que o simulador apresentado possui metade da resolução de um olho humano. Estação de pilotagem: o uso de uma estação de pilotagem em vez de um veículo adaptado altera a sensação de realismo, uma vez que os comandos e o campo de visão do simulador não são iguais aos de um veículo real.

Falta de impactos para o condutor: o ambiente virtual elimina possíveis impactos financeiros e/ou físicos como resultados de um acidente. Isso pode afetar o comportamento do condutor, que pode dirigir de forma mais agressiva e assumir maiores riscos, divergindo de seu comportamento real. 


\section{METODOLOGIA ADOTADA}

0 trabalho de modelagem da via e seu entorno foi um trabalho conjunto entre pesquisadores da Universidade de São Paulo e engenheiros da iniciativa privada. Inicialmente, foi feito um mapeamento de um trecho de dez quilômetros de extensão de uma rodovia federal brasileira de interesse, com auxílio de plataforma inercial. Foram coletadas, a cada cinco metros, latitude e longitude e inclinações longitudinal e lateral. 0 erro de medição das inclinações foi de $0,12^{\circ}$. Juntamente às medições, foram realizadas filmagens do entorno para posterior utilização na montagem do cenário. Informações adicionais foram obtidas nos desenhos de projeto da rodovia e os desenhos foram fornecidos pela concessionária responsável pela administração do trecho.

Após tratamento dos dados coletados, eles serviram como dados de entrada para a construção da geometria da via no ROD, módulo do $\mathrm{VTD}^{\circledR}$. Com base nas filmagens e em fotos disponíveis na internet, o entorno foi inserido (construções, acidentes geográficos, vegetação). A última etapa foi a inclusão da sinalização existente, com base em desenhos técnicos fornecidos pela concessionária responsável pela administração do trecho. A Figura 4 mostra uma imagem de um mesmo trecho nos dois ambientes: (a) real e (b) virtual. A atividade de levantamento de dados e modelagem da rodovia demorou quatro meses.

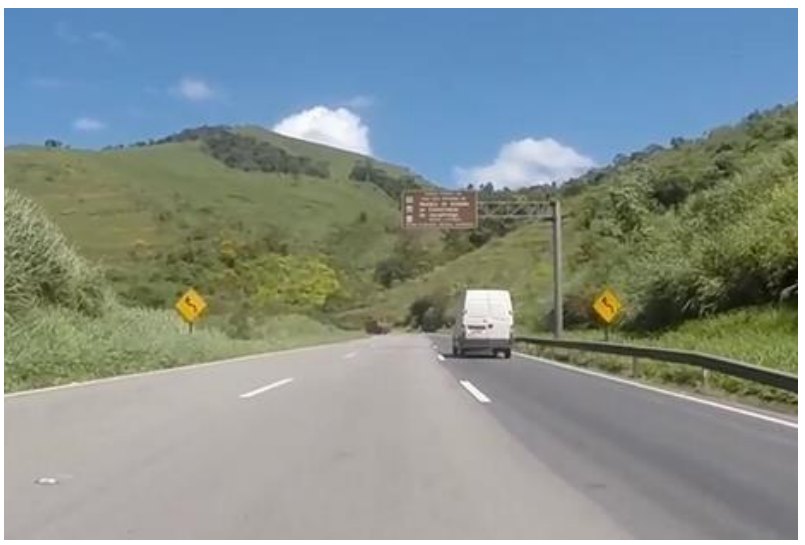

(a)

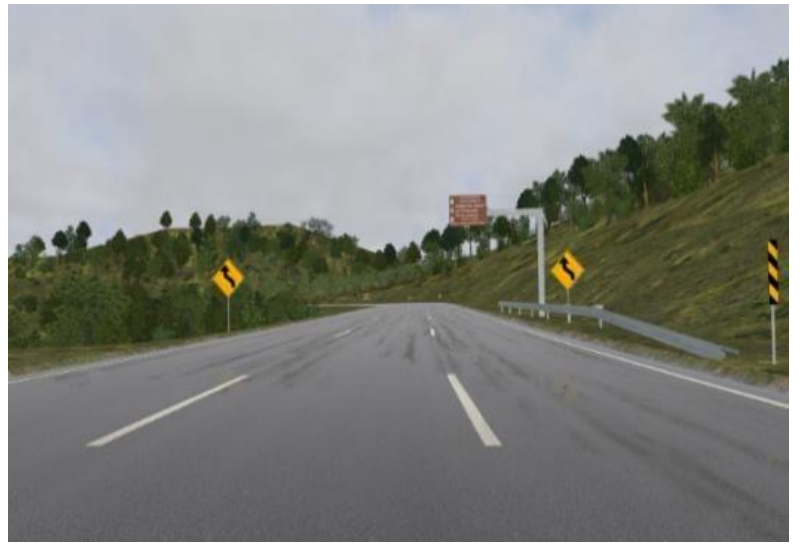

(b)

Figura 4. Modelagem da rodovia: (a) cenário real (b) cenário virtual

O rastreamento ocular permite o estudo do comportamento dos condutores, bem como a informação que o condutor está processando e a complexidade desse processamento (Fisher et al, 2011). Na literatura, existem duas técnicas de monitoramento do rastreamento ocular: medição dos olhos com relação à cabeça do condutor e medição da orientação dos olhos no espaço (Duchowski, 2007). 0 equipamento de rastreio ocular do simulador utiliza a técnica de monitoramento por meio da medição da orientação dos olhos no espaço. Por esse motivo, é necessária uma calibração do equipamento sempre que há troca do condutor, ou sempre que ele move a cabeça lateralmente.

A proximidade entre os comportamentos dos veículos virtual e real é vital para a obtenção de correlações entre resultados coletados no campo e em simuladores. A percepção de efeitos, como limites de manobrabilidade, sensibilidade à velocidade, variação do "peso" (torque) do volante e subesterço, deve ser o mais próximo possível da realidade para que sejam obtidos resultados coerentes quando um simulador é utilizado.

A modelagem do veículo foi feita utilizando metodologia de sistemas multicorpos, que consiste em um conjunto de elementos interconectados por meio de juntas e sujeitos a vínculos. Esses sistemas mecânicos respondem a esforços e movimentos pré-estabelecidos. A Figura 5 ilustra um modelo de simplificado para representação em multicorpos.

As equações resultantes dessa teoria são complexas e demandam tempo elevado para solução. Os sistemas em tempo real necessitam de uma modelagem simplificada, que pode ser feita por meio das 
equações ou do modelo físico do veículo. Costa Neto (1992) propõe uma simplificação na geometria da suspensão que resulta em modelos fidedignos cujas equações conseguem ser resolvidas em tempo real.

O programa utilizado para a modelagem veicular gera as equações de movimento para análise dinâmica do sistema modelado e usa o método de Newton-Euler Lagrange para construção dos sistemas de equações. Tais equações são resolvidas em tempo real, gerando a resposta do veículo às entradas impostas pelo condutor ou por manobras pré-estabelecidas.

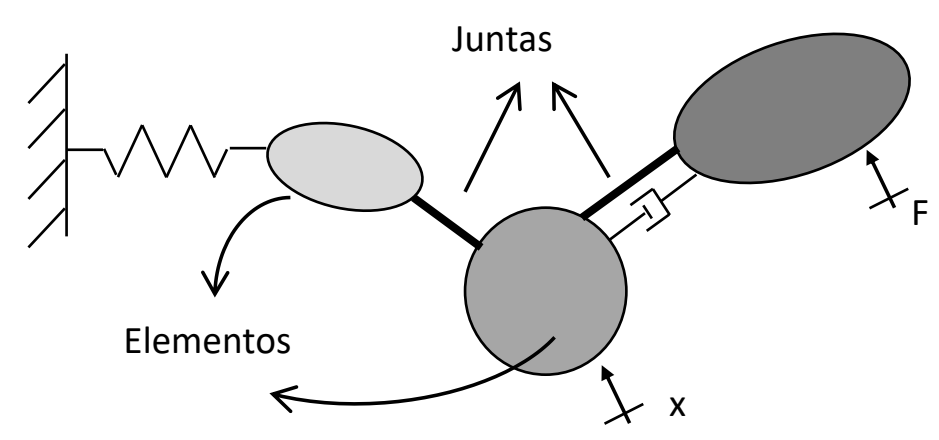

Figura 5. Modelo em multicorpos (Adaptado de Costa Neto, 1992)

Na modelagem, são utilizados parâmetros reais do veículo, tais como curvas de rigidez de mola, amortecimento e torque do motor, relação de transmissão, eficiência de frenagem, modelagem do pneumático, dentre outros. Após a finalização da construção do modelo, ele passa por um processo de validação. Os resultados obtidos das análises com o modelo matemático são comparados aos resultados observados em manobras similares realizadas com protótipos físicos.

O modelo em multicorpos utilizado foi construído por engenheiros da PSA Peugeot Citroën Brasil ${ }^{\circledR} \mathrm{e}$ representa um veículo compacto $\mathrm{C} 3$, com câmbio automático (com possibilidade de alteração para câmbio mecânico), motor 1000 cc e direção elétrica.

0 modelo veicular foi cedido pela iniciativa privada. Estima-se que para uma modelagem com nível de detalhe e representatividade similar sejam necessários de 8 a 12 meses de trabalho de um engenheiro com conhecimento na área de simulações em multicorpos.

\section{APLICAÇÕES DO SIMULADOR - ESTUDOS DE CASO}

A seguir são apresentados dois estudos realizados no simulador descrito:

- Análise da percepção da sinalização vertical rodoviária em ambientes simulados de direção (Rangel, 2015);

- Análise da fixação ocular de condutores através de teste de carga mental (Vieira et al, 2015).

\subsection{Análise da percepção da sinalização vertical rodoviária em ambientes simulados de direção}

Rangel (2015) analisou como condutores percebem a sinalização vertical de uma rodovia em um ambiente virtual. Vinte e um voluntários dirigiram separadamente em um trecho de dez quilômetros no simulador. As variáveis monitoradas foram: distância de percepção, número de visualizações por placa, e tempo de observação para cada um dos trinta e um sinais de trânsito existentes no trecho. Ao final, foi apresentado um projeto de sinalização vertical que melhora a percepção dos condutores e aumenta a quantidade de condutores que visualizam a sinalização e o tempo de fixação do olhar deles na sinalização.

Um resumo das análises estatísticas realizadas no estudo com o simulador é apresentado na Tabela 1. Algumas das análises foram feitas baseadas nas respostas dos voluntários a um questionário aplicado previamente, com o objetivo de caracterizar a amostra selecionada (idade, gênero, tipo de condutor). As 
variáveis independentes da análise desagregada eram informações conhecidas e utilizadas na modelagem do cenário; enquanto as variáveis dependentes de ambas as análises eram informações coletadas ou medidas durante os eventos simulados. Além disso, as variáveis independentes da análise agregada possuíam informações conhecidas e informações obtidas a partir do experimento.

Tabela 1: Resumo das análises estatísticas realizadas

\begin{tabular}{|c|c|c|c|}
\hline \multicolumn{2}{|c|}{$\begin{array}{l}\text { Análise desagregada } \\
\end{array}$} & \multicolumn{2}{|c|}{$\begin{array}{l}\text { Análise agregada } \\
\end{array}$} \\
\hline Variável dependente & Variável Independente & Variável dependente & Variável Independente \\
\hline $\begin{array}{l}\text { Probabilidade de percepção } \\
\text { do sinal }\end{array}$ & $\begin{array}{l}\text { Tipo de sinal } \\
\text { Duplicidade } \\
\text { Proximidade entre sinais } \\
\text { Posicionamento } \\
\text { Velocidade do veículo }\end{array}$ & $\begin{array}{l}\text { Velocidade de operação } \\
\text { no simulador }\end{array}$ & $\begin{array}{l}\text { Velocidade real de operação no } \\
\text { trecho }\end{array}$ \\
\hline Número de fixações & $\begin{array}{l}\text { Tipo de sinal } \\
\text { Proximidade entre sinais } \\
\text { Duplicidade }\end{array}$ & \multirow{2}{*}{$\begin{array}{l}\text { Velocidade média } \\
\text { espacial }\end{array}$} & $\begin{array}{l}\text { Idade } \\
\text { Gênero } \\
\text { Tipo de condutor }\end{array}$ \\
\hline Tempo de observação & $\begin{array}{l}\text { Tipo de sinal } \\
\text { Proximidade entre sinais } \\
\text { Duplicidade }\end{array}$ & & $\begin{array}{l}\text { Variância no ângulo de direção do } \\
\text { volante } \\
\text { Curvatura do traçado }\end{array}$ \\
\hline Distância de percepção & $\begin{array}{l}\text { Tipo de sinal } \\
\text { Proximidade entre sinais } \\
\text { Duplicidade } \\
\end{array}$ & \multirow{2}{*}{$\begin{array}{l}\text { Porcentagem de sinais } \\
\text { percebidos }\end{array}$} & $\begin{array}{l}\text { Idade } \\
\text { Gênero } \\
\text { Tipo de condutor }\end{array}$ \\
\hline Variação da velocidade & $\begin{array}{l}\text { Tipo de sinal } \\
\text { Proximidade entre sinais } \\
\text { Duplicidade }\end{array}$ & & $\begin{array}{l}\text { Limitações visuais } \\
\text { Velocidade média } \\
\text { Variância no ângulo de direção do } \\
\text { volante }\end{array}$ \\
\hline
\end{tabular}

Por ser um ambiente virtual, onde a frequência de aquisição de dados não gera ônus ao pesquisador, foi possível extrair informação como a apresentada na Figura 6. A escala de cores representa diferentes tipos de placas. No eixo das abscissas estão as localizações de cada placa ao longo do trecho e no eixo das coordenadas está a porcentagem de condutores que perceberam a sinalização.

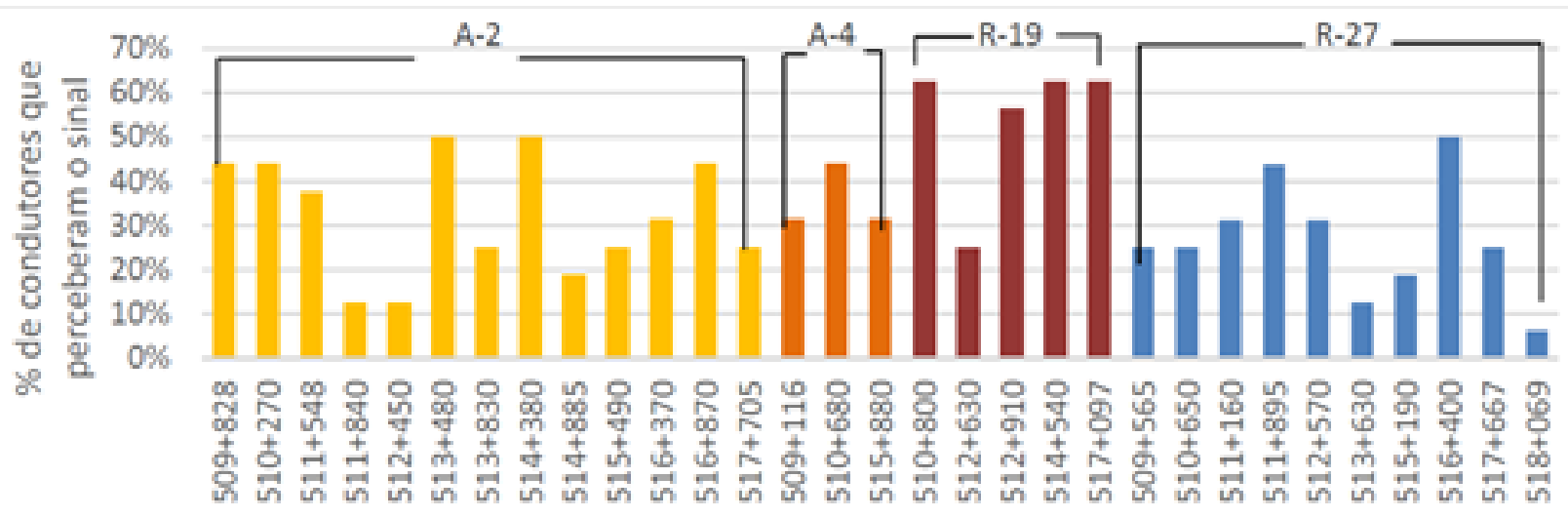

Figura 6. Porcentagem de condutores que perceberam cada placa (Rangel, 2015)

A percepção da sinalização foi feita com o uso do equipamento de rastreio ocular Smart Eye ${ }^{\circledR}$ e o programa Mapps ${ }^{\circledR}$. A Figura 7 mostra como foi determinada a deteç̧ão de placas pelos condutores. 0 círculo verde na placa de sinalização indica a área o condutor olhou naquele instante. A taxa de aquisição definida foi de 0,016 segundos.

A distribuição das distâncias de reação à sinalização também foi analisada no estudo (Figura 8). Essa informação permitiu a definição das posições ideais para cada sinalização.

A Figura 9 ilustra o diagrama de caixas da porcentagem de condutores que perceberam cada sinal 
segundo seu tipo. 0 sinal R-19 foi o mais percebido no simulador, com uma porcentagem média de percepção de $54 \pm 16 \%$. Em sequência, a sinalização A-4 obteve percepção de $35 \pm 7 \%$, semelhante à da sinalização A-2 com $32 \pm 13 \%$. Foi o sinal R-27 que obteve o menor percentual de percepção: $27 \pm 13$ \%. Tal ordem coincide com a hierarquia ou relevância que deveriam ter esses sinais para os condutores de veículos leves.

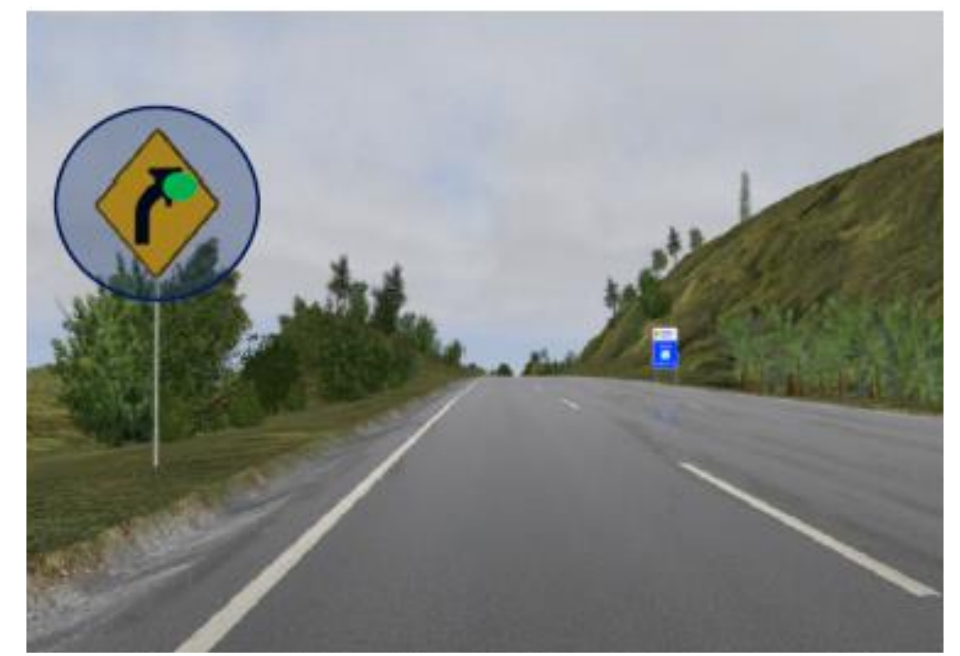

Figura 7. Detecção de placa no simulador (Rangel, 2015)

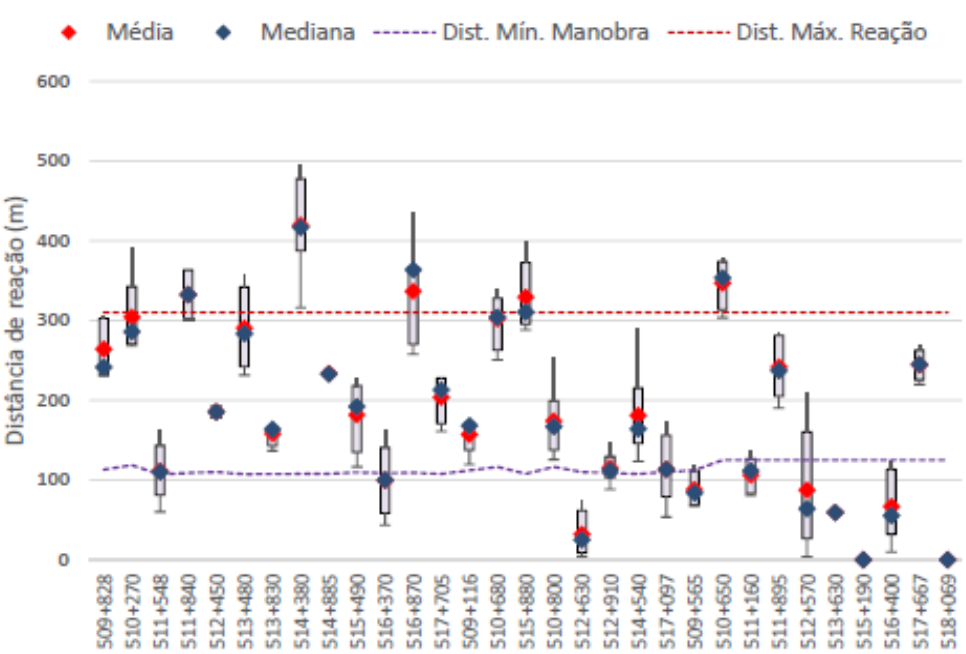

Figura 8. Distâncias de reação dos condutores para cada placa (Rangel, 2015)

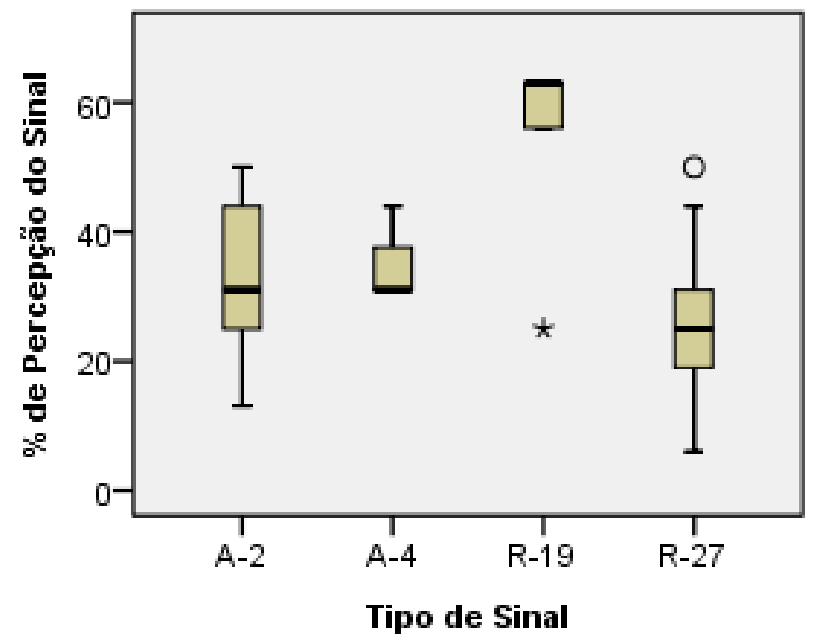

Figura 9. Porcentagem de percepção das placas, por tipo de placa (Rangel, 2015) 
De forma geral, o resultado obtido por Rangel (2015) seguiu àquele reportado pela literatura: somente as placas de limite de velocidade afetaram o comportamento dos condutores; o tempo de observação da sinalização foi de 360 milésimos de segundos; somente uma a cada três placas eram notadas; e a distância de percepção foi de $100 \mathrm{~m}$. Com isso, foi possível propor medidas que aprimorassem a sinalização existente no trecho da rodovia estudada.

\subsection{Análise da fixação ocular de condutores através de teste de carga mental}

Viera et al (2015) conduziram, no mesmo simulador, um estudo com o objetivo de comparar o comportamento de condutores em situação normal de concentração e com uma carga mental adicional.

A carga mental consistiu em uma adaptação do teste Pacing Auditory on a Serial Addition Task (PASAT). Durante a atividade de condução, fez-se uma apresentação oral periódica de números e o participante deveria retornar a soma dos últimos numerais dentro de um intervalo de tempo. 0 objetivo da carga mental foi avaliar a perda da capacidade cognitiva do condutor ao desviar a atenção da atividade principal (direção) para uma tarefa secundária (resultado da soma de números).

A comparação foi feita com base no tempo que cada condutor fixava o olhar em um determinado ponto durante o evento simulado. 0 trajeto a ser percorrido pelos condutores foi dividido em três trechos, definidos com base no tempo de andamento do teste e não na localização do trecho em que o condutor se encontrava. 0 condutor tinha liberdade para dirigir na velocidade que considerasse pertinente. A Tabela 2 ilustra os tempos pré-definidos para cada trecho. No caso do trecho 3, o tempo de duração foi o necessário para que o condutor chegasse ao final do trajeto. Cada condutor executou duas passagens para coleta de dados.

Tabela 2: Definição dos trechos (Vieira et al, 2015)

\begin{tabular}{lcc}
\hline & Duração [s] & Caracterização \\
\hline Trecho 1 & 60 & Fase pré-teste, sem carga mental \\
Trecho 2 & 180 & Fase teste, com carga mental \\
Trecho 3 & Indeterminado & Fase pós-teste, sem carga mental \\
\hline
\end{tabular}

A Figura 10 exemplifica como foi feito o rastreio ocular durante o experimento. É possível identificar as três áreas onde o condutor fixou o olhar (círculos amarelos).

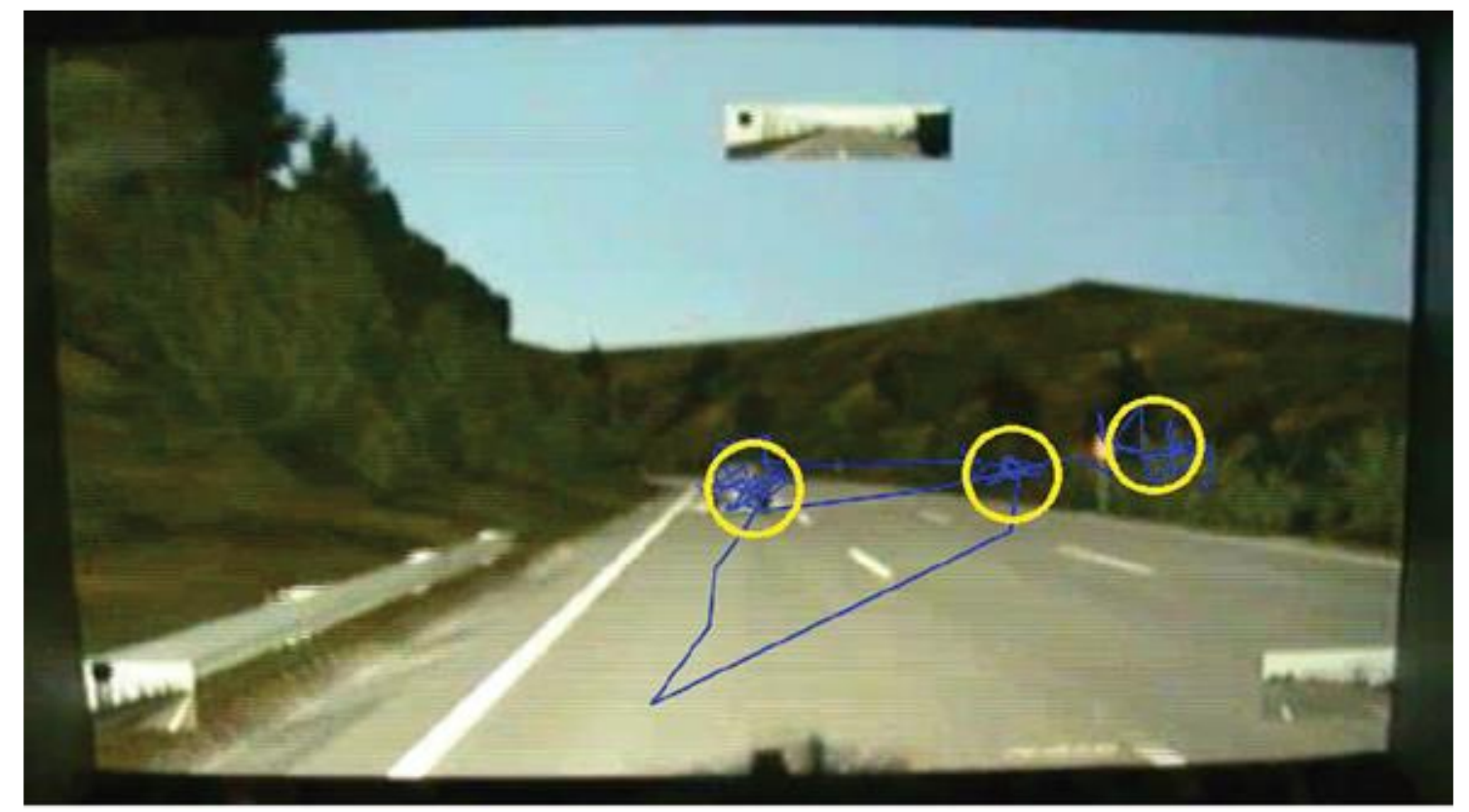

Figura 10. Rastreio ocular (Vieira et al, 2015) 
O tempo de fixação do olhar de cada condutor com e sem carga mental foi comparado individualmente, sem comparações entre condutores (Tabela 3). 0 Smart Eye ${ }^{\circledR}$ e o programa Mapps ${ }^{\circledR}$ (pós-processamento dos dados) foram utilizados para a coleta das informações.

Tabela 3: Tempo de fixação do olhar dos condutores por evento (Vieira et al, 2015)

\begin{tabular}{lcccccc}
\hline & \multicolumn{2}{c}{ TRECHO 1 } & \multicolumn{2}{c}{ TRECHO 2 } & \multicolumn{2}{c}{ TRECHO 3 } \\
& Sem Carga & Com Carga & Sem Carga & Com Carga & Sem Carga & Com Carga \\
\hline Condutor 1 & 59 & 55 & 195 & 180 & 201 & 201 \\
Condutor 2 & 50 & 55 & 170 & 180 & 349 & 311 \\
Condutor 3 & 46 & 55 & 163 & 180 & 132 & 153 \\
Condutor 4 & 33 & 55 & 177 & 180 & 136 & 114 \\
Condutor 5 & 54 & 55 & 171 & 180 & 253 & 247 \\
Condutor 6 & 50 & 55 & 205 & 180 & 203 & 182 \\
Condutor 7 & 53 & 55 & 188 & 180 & 204 & 190 \\
Condutor 8 & 48 & 55 & 172 & 180 & 105 & 111 \\
Condutor 9 & 55 & 55 & 197 & 180 & 109 & 4 \\
Condutor 10 & 54 & 55 & 162 & 180 & 323 & 293 \\
Condutor 11 & 43 & 55 & 227 & 180 & 212 & 171 \\
Condutor 12 & 50 & 55 & 156 & 180 & 132 & 101 \\
Condutor 13 & 58 & 55 & 189 & 180 & 343 & 338 \\
Condutor 14 & 63 & 55 & 246 & 180 & 255 & 191 \\
Condutor 15 & 49 & 55 & 184 & 180 & 110 & 145 \\
\hline
\end{tabular}

Após tratar e analisar estatisticamente os dados, os pesquisadores concluíram que o teste proposto não apresentou significância estatística para mostrar diferenças no comportamento dos condutores com a frequência utilizada para aplicação da tarefa secundária (carga mental). Eles também mostram que há uma variação entre as velocidades médias observadas no experimento e as velocidades médias coletadas em campo por Rangel (2015), devido às limitações do simulador de direção.

\section{CONCLUSÕES}

Originalmente, os simuladores de direção eram aplicados pela engenharia da indústria automotiva para ajustes finos de suspensão. Juntamente ao avanço tecnológico, pesquisadores de áreas diversas, outras engenharias, psicologia e até mesmo medicina, fizeram uso dos simuladores para ampliarem seus conhecimentos. Por ser uma ferramenta durável e permitir usos simultâneos, os simuladores são ferramentas de alto valor agregado a um custo relativamente baixo quando comparados aos investimentos necessários para realização de experimentos naturalísticos.

Dentre as vantagens do uso de simuladores, podem-se citar: reprodução de situações de risco sem oferecer risco à integridade física de voluntários e pesquisadores; redução do tempo dos experimentos; aumento da amplitude dos experimentos; controle experimental; e redução nos custos frente aos experimentos realizados em campo.

As principais desvantagens observadas pelo uso do simulador foram: a falta de consequência física, no caso de um acidente, pode interferir em alguns casos; o ambiente virtual não tem impacto financeiro para o condutor, o que pode impactar seu comportamento; o fato de se trabalhar com uma base fixa pode aumentar a dispersão dos resultados devido à falta das acelerações a que o condutor fica submetido; e a falta das acelerações, que também pode causar enjoos em algumas pessoas, o que pode resultar em um aumento de tempo do experimento. A necessidade de modelos veiculares fidedignos, exigindo mão de obra especializada, é outra desvantagem do equipamento.

Apesar das limitações apresentadas, o uso dos simuladores de direção traz grandes contribuições para estudos com interesse em avaliar condutores, veículos e vias (geometria ou entornos). Apesar de necessitar de investimento financeiro inicial alto, possui uso mais amplo em comparação às despesas que cercam estudos em campo. 


\section{REFERÊNCIAS}

Ahlström, C., Dukic, T., IvarssoN, E., Kircher, A., Rydbeck, B., Viström, M. (2010). Performance of a one-camera and a three camera system: ViP publication. Disponível em: <http://smarteye.se/wp-content/uploads/2015/01/Ahlstrom-Christer-et.al..pdf $\geq$.

Balci, O. (1994). Validation, verification, and testing techniques throughout the life cycle of a simulation study. Annals of Operations Research, v. 53, n. 1-4, p. 121-173. DOI: 10.1007/BF02136828

Bella, F. (2007). Parameters for evaluation of speed differential: contribution using driving simulator. transportation research record. Journal of the Transportation Research Board, v. 2023, p. 37-43. DOI: 10.3141/2023-05.

Bella, F.; Calvi, A. (2013). Effects of simulated day and night driving on the speed differential in tangent-curve transition: a pilot study using driving simulator. Traffic Injury Prevention, v. 14, n. 4, p. 413-423. DOI: 10.1080/15389588.2012.716880.

Blana, E. (1996a). Driving simulator validation studies: a literature review leeds. UK: Institute of Transport Studies, University of Leeds, Working Paper 480. Disponível em: < http://eprints.whiterose.ac.uk/2111/ >.

Blana, E. (1996b). A survey of driving research simulators around the world. UK: Institute of Transport Studies, University of Leeds, Working Paper 481. Disponível em: < http://eprints.whiterose.ac.uk/2110/ >

Boyle, L. N. e J. D. Lee (2010). Using driving simulators to assess driving safety. Accident Analysis and Prevention, v 42, p 787788. DOI: $10.1016 /$ j.aap.2010.03.006.

Broughton, K. L.; Switzer, F.; Scott, D. (2007). Car following decisions under three visibility conditions and two speeds tested with a driving simulator. Accident Analysis \& Prevention, v. 39, n. 1, p. 106-116. DOI: 10.1016/j.aap.2006.06.009.

Costa Neto, A. (1992). Application of multibody system techniques to automotive vehicle chassis simulation for motion control studies. Tese (Doutorado em Engenharia Mecânica) - University of Warwick. Disponível em: < https://www.resear chgate.net/publication/266315563_Application_of_Multibody_Systems_Techniques_to_Automotive_Vehicle_Chassis_Simulation_for_Motion_Control_Studies>

Duchowski, A. (2007). Eye tracking methodology, theory and practice. 2 ed. Londres: Springer. DOI: 10.1007/978-1-84628609-4.

Devlin, A. et al. (2012). Investigating driving behaviour of older drivers with mild cognitive impairment using a portable driving simulator. Accident Analysis \& Prevention, v. 49, p. 300-307, 11. D0I: 10.1016/j.aap.2012.02.022.

Evgenikos, P. et al. (2010). WHO | Data systems: a road safety manual for decision-makers and practitioners. WHO. Disponível em: < http://www.who.int/roadsafety/projects/manual/data/en/>.

Filtness, A. J. et al. (2017). Sleep-related eye symptoms and their potential for identifying driver sleepiness. Journal of Sleep Research, v. 23, n. 5, p. 568-575. DOI: 10.1111/jsr.12163.

Fernandes, C. G. (2009). Método probabilístico para o estudo de sistemas dinâmicos não-lineares: uma aplicação em dinâmica veicular. Tese (Doutorado em Engenharia Naval e Oceânica) - Escola Politécnica, Universidade de São Paulo. DOI: 10.11606/T.3.2009.tde-21122009-133620.

Fisher, D. L. et al (2011). Handbook of driving simulation for engineering, medicine, and psychology. Boca Raton, USA: CRC Press.

Godley, S. T.; Triggs, T. J.; Fildes, B. N. (2002). Driving simulator validation for speed research. Accident Analysis \& Prevention, v. 34, n. 5, p. 589-600, 9. DOI: 10.1016/S0001-4575(01)00056-2.

Greenberg, J. B. (2011). Physical fidelity of driving simulators. In; Fisher, D. I. et al. Handbook of driving simulation for engineering, medicine, and psychology. Boca Raton, USA: CRC Press.

Helland, A. et al. (2013). Comparison of driving simulator performance with real driving after alcohol intake: A randomized, single blind, placebo-controlled, cross-over trial. Accident Analysis and Prevention, v 53, p 9-16. DOI: 10.1016/j.aap.2012.12.042.

Jamson, S; Lai, F.; Jamson, H. (2010). Driving simulator for robust comparisons: a case study evaluating road safety engineering treatments. Accident Analysis and Prevention, v 42, n. 3, p 961-971. DOI: 10.1016/j.aap.2009.04.014.

Källhammer, J.-E. et al. (2007). Shouldn't cars react as drivers expect?, Proceedings of the fourth international driving symposium on human factors in driver assessment, training and vehicle design. autoliv research. Vårgårda, Sweden, 2007. Disponível em: < https://www.researchgate.net/profile/Jan-Erik_Kaellhammer/publication/242150024_SHOULDN'T_CARS_REACT_AS_DRIVERS_EXPECT/links/00b4951d5db1e8ad5f000000.pdf >

Kemeny, A.; Panerai, F. (2003). Evaluating perception in driving simulation experiments. Trends in Cognitive Sciences, v. 7, n. 1, p. 31-37. DOI: 10.1016/S1364-6613(02)00011-6.

Knapper, A. Christoph, M., Hagenzieker, M., Brookhuis, K. (2015) Comparing a driving simulator to the real road regarding distracted driving speed. European Journal of Transport and Infrastructure Research, v. 2, n. 15, p. 205-225. Disponível em: < http://repository.tudelft.nl/islandora/object/uuid:de0f6373-5856-45bc-9af4-fa74c6521997?collection=research>.

Lee, H.C.; Cameron, D.; Lee, A. H. (2003). Assessing the driving performance of older adult drivers: on-road versus simulated driving. Accident Analysis \& Prevention, v. 35, n. 5, p. 797-803. DOI: 10.1016/S0001-4575(02)00083-0.

Rangel, M.A.C. (2015). Análise da percepção da sinalização vertical por parte do condutor, utilizando ambientes simulados de direção: um estudo de caso na rodovia BR-116. Dissertação (Mestrado em Engenharia de Transporte e Infraestrutura de Transportes) - Escola de Engenharia São Carlos, Universidade de São Paulo. DOI: 10.11606/D.18.2015.tde-29072015101734.

Reymond, G. et al. (2001). Role of lateral acceleration in curve driving: driver model and experiments on a real vehicle and a driving simulator. Human Factors, v. 43, n. 3, p. 483-495. DOI: 10.1518/001872001775898188. 
Rumschlag, G. et al. (2015). The effects of texting on driving performance in a driving simulator: the influence of driver age. Accident Analysis \& Prevention, v. 74, n. 6, p. 145-149. DOI: 10.1016/j.aap.2014.10.009.

Törnros, J. (1998). Driving behaviour in a real and a simulated road tunnel: a validation study. Accident Analysis \& Prevention, v. 30, n. 4, p. 497-503, 7. DOI: 10.1016/S0001-4575(97)00099-7.

Underwood, G.; Crundall; D., Chapman, P. (2011). Driving simulator validation with hazard perception. Transportation Research Part F: Traffic Psychology and Behavior, v. 14, n. 6, p. 435-446. DOI: 10.1016/j.trf.2011.04.008.

Vieira, F. S. et al. (2015). Análise da fixação ocular de condutores através de teste de carga mental. XXIX Congresso Nacional de Pesquisa em Transporte da ANPET - Ouro Preto / MG, 2015. Disponível em: < http://www.anpet.org.br/xxixanpet/anais/documents/AC838.pdf >.

WHO | World Health Organization (2015). Global status report on road safety: time for action. Geneva: WHO. Disponível em: <http://www.who.int/violence_injury_prevention/road_safety_status/2015/en/>. Acesso em 16 jul. 2015.

Yan, X. et al. (2008) Validating a driving simulator using surrogate safety measures. Accident Analysis \& Prevention, v. 40, n. 1, p. 274-288. DOI: 10.1016/j.trf.2011.04.008. 